\title{
RNA Binding Proteins Control Transdifferentiation of Hepatic Stellate Cells into Myofibroblasts
}

\author{
Sihyung Wang ${ }^{\mathrm{a}, \mathrm{c}}$ Youngmi Jung ${ }^{\mathrm{a}, \mathrm{c}, \mathrm{d}}$ Jeongeun Hyun ${ }^{\mathrm{a}} \quad$ Matthew Friedersdorf ${ }^{\mathrm{b}}$ \\ Seh-Hoon Oh ${ }^{\mathrm{a}}$ Jieun Kim ${ }^{\mathrm{c}}$ Richard T. Premont ${ }^{\mathrm{a}}$ Jack D. Keene ${ }^{\mathrm{b}}$ \\ Anna Mae Diehla \\ aDepartment of Medicine, Duke University, Durham, bepartment of Molecular Genetics and \\ Microbiology, Duke University, Durham, USA, 'Department of Integrated Biological Science, Pusan \\ National University, Pusan, ${ }^{d}$ Department of Biological Sciences, Pusan National University, Pusan, South \\ Korea
}

\section{Key Words}

RNA binding protein - Insulin-like growth factor 2 binding protein 3 - IMP3 - Cirrhosis • Mesenchymal stem cells (MSCs) - Epithelial-mesenchymal transition (EMT) • Transforming growth factor beta (TGF- $\beta$ ) • MicroRNA (miRNA)

\begin{abstract}
Background/Aims: Myofibroblasts (MF) derived from quiescent nonfibrogenic hepatic stellate cells (HSC) are the major sources of fibrous matrix in cirrhosis. Because many factors interact to regulate expansion and regression of MF-HSC populations, efforts to prevent cirrhosis by targeting any one factor have had limited success, motivating research to identify mechanisms that integrate these diverse inputs. As key components of RNA regulons, RNA binding proteins (RBPs) may fulfill this function by orchestrating changes in the expression of multiple genes that must be coordinately regulated to affect the complex phenotypic modifications required for HSC transdifferentiation. Methods: We profiled the transcriptomes of quiescent and MF-HSC to identify RBPs that were differentially-expressed during HSC transdifferentiation, manipulated the expression of the most significantly induced RBP, insulin like growth factor 2 binding protein 3 (Igf2bp3), and evaluated transcriptomic and phenotypic effects. Results: Depleting Igf $2 \mathrm{bp} 3$ changed the expression of thousands of HSC genes, including multiple targets of TGF- $\beta$ signaling, and caused HSCs to reacquire a less proliferative, less myofibroblastic phenotype. RNA immunoprecipitation assays demonstrated that some of these effects were mediated by direct physical interactions between Igf $2 \mathrm{bp} 3$ and mRNAs that control proliferative activity and mesenchymal traits. Inhibiting TGF- $\beta$ receptor- 1 signaling revealed a microRNA-dependent mechanism that induces Igf $2 \mathrm{bp} 3$. Conclusions: The aggregate results indicate that HSC transdifferentiation is ultimately dictated by Igf2bp3dependent RNA regulons and thus, can be controlled simply by manipulating Igf2bp3.

S. Wang and Y. Jung contributed equally to this work.

\begin{tabular}{ll}
\hline Anna Mae Diehl, MD & Snyderman Building, Suite 1073, Duke University, Durham, NC 27710 (USA) \\
Tel. 1-919-684-2366, E-Mail annamae.diehl@duke.edu
\end{tabular} 


\section{Introduction}

Cirrhosis, the endpoint of liver damage caused by various liver diseases, is already a major cause of death worldwide, and is becoming more prevalent due to emerging epidemics of chronic alcohol- and obesity-related liver disease. Currently, there are no effective drug approaches to prevent or reverse cirrhosis in individuals with ongoing liver injury. Because liver transplantation is a viable option for only a minority of these patients, new preventative and therapeutic approaches for cirrhosis are desperately needed [1]. Success will require improved understanding of fundamental mechanisms that control cirrhosis pathogenesis.

Cirrhosis results from progressive replacement of functional liver parenchyma by fibrous scar. Fibrosis is driven by myofibroblasts (MF) that accumulate as part of wound healing responses that are triggered by tissue injury. Most of the MF in chronically injured livers derive from hepatic stellate cells (HSC) that are quiescent (i.e., not proliferative or fibrogenic) in healthy livers [2, 3]. MF-HSCs have many roles in wound healing. In addition to being major sources of fibrous matrix, MF-HSCs generate factors that regulate hepatocyte proliferation and viability, modulate immune cell recruitment to the damaged liver, control remodeling of the hepatic vasculature, and mediate the growth and differentiation of progenitor populations [4]. These functions are critical for the regeneration of healthy hepatic parenchyma as evidenced by the fact that preventing MF accumulation blocks regeneration of the liver after partial hepatectomy [5]. Importantly, MF accumulation is a transient phase of effective liver regeneration, while cirrhosis is characterized by persistence of large numbers of MF-HSC [3]. Hence, mechanisms that control the size of the hepatic MF-HSC population dictate whether or not liver tissue is regenerated during injury or progressively replaced by scar. Delineating these mechanisms will identify tractable targets to optimize effective liver regeneration and thus, prevent cirrhosis.

We evaluated the hypothesis that RNA binding proteins (RBPs) orchestrate critical fate decisions in HSCs to control the size of the hepatic MF-HSC population. RBPs link the cellular transcriptome and proteome by directly binding to discrete motifs on specific RNA molecules in order to coordinate the localization, stability, and translation of families of mRNAs with related RBP binding sites. As such, RBPs refine the actions of microRNAs and long noncoding RNAs, together forming RNA regulons that orchestrate changes in the expression of multiple genes that must be coordinately regulated to affect complex phenotypic changes, such as proliferation and differentiation [6]. The significance of RBPs as cell fate regulators is exemplified by evidence that they are highly evolutionarily conserved in stem-like cells from sea urchins to mammals [7]. Although the role of RBPs in HSC biology is virtually unstudied, this level of regulation is likely to be important because HSC are liver-resident members of a ubiquitous mesenchymal stem cell-like pericyte population [8]. Further, it is well established that the inherent plasticity of HSC enables them to undergo reversible reprogramming in response to liver injury $[9,10]$. For example, enrichment of the microenvironment with TGF- $\beta$, an injury-induced fibrogenic factor, stimulates Q-HSC to acquire a proliferative myofibroblastic phenotype, and some of these MF-HSC revert back to the basal, more quiescent, phenotype as tissue injury resolves and TGF- $\beta$ levels dissipate [11]. We propose that the size and activity of the MF-HSC population can be controlled therapeutically during liver injury by targeting RBPs that regulate HSC differentiation.

Herein, we report the results of studies that revealed that expression of specific RBPs changes significantly as Q-HSC differentiate to become MF-HSC, both in vitro and in vivo. Importantly, several of these differentially expressed RBPs have been reported to regulate epithelial-to-mesenchymal transitions and mesenchymal-to-epithelial transitions (EMT/MET) in other cell types with high phenotypic plasticity, including stem-like cells. Unexpectedly, we discovered that silencing a single RBP in HSC was sufficient to alter steady state mRNA levels of thousands of genes, and subsequent gene enrichment analysis revealed that this particular RBP is a master regulator of TGF- $\beta$ signaling, an acknowledged driver of myofibroblastic differentiation in HSC.

\section{KARGER}




\section{Cellular Physiology Cell Physiol Biochem 2018;48:1215-1229

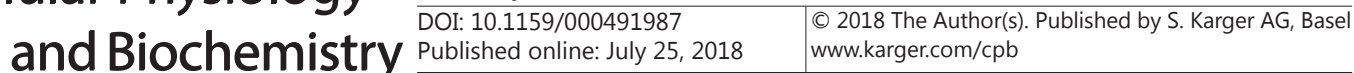

Wang et al.: RNA Binding Proteins in Stellate Cell Activation

\section{Materials and Methods}

\section{Mouse HSC Isolation}

Primary HSC were isolated from WT mice using standard approaches [12]. Cells were pooled from at least 6 mice for each experiment (total $n \geq 40$ mice) and all experiments were repeated at least three times to assure data reproducibility. Briefly, after in situ perfusion of the liver with pronase (Roche) followed by collagenase D (Roche), dispersed cell suspensions were layered on a discontinuous density gradient of 8.2 and $15.6 \%$ Histodenz (Sigma-Aldrich). The resulting upper layer consisted of $>96 \%$ HSCs. The viability of all cells was verified by phase contrast microscopy and propidium iodide exclusion. The viability of all cells utilized for culture was $>98 \%$. Isolated HSC were seeded at a density of $3 \times 10^{2}$ cells $/ \mu \mathrm{m}^{2}$ in DMEM (Invitrogen) supplemented with 10\% FBS, streptomycin and penicillin, and non- adherent cells and debris were rinsed away $2 \mathrm{~h}$ after initial plating.

\section{Animal Models}

Residual frozen liver tissues from previously reported experiments in bile duct ligated (BDL) rats with and without Roux-en-Y biliary-intestinal anastomosis and sham-operated controls [13] were processed to obtain RNA for qRT PCR analysis. Fibrotic livers of male mice treated with $\mathrm{CCl}_{4}$ for 6 or 10 weeks were obtained as previously described [14]. Briefly, C57BL/ 6 mice at 7 weeks old received carbon tetrachloride $\left(\mathrm{CCl}_{4}, 0.6 \mathrm{ml} / \mathrm{kg}\right.$ body weight $)$ or corn oil by intraperitoneal injection twice a week for $6(\mathrm{n}=5)$ or 10 weeks $(\mathrm{n}=6)$. Animal care and surgical procedures were approved by the Duke University and Pusan National University Institutional Animal Care and Use Committees, and carried out in accordance with the provisions of the NRC Guide for the Care and Use of Laboratory Animals.

\section{Human Subjects}

De-identified frozen tissues from percutaneous liver biopsies of 11 NAFLD (fibrosis stage $0, n=3 / 1-2$, $n=5 / 3-4, n=3$ ) patients with histologically staged fibrosis were obtained from the Duke University School of Medicine Tissue Bank Shared Resource and studied in accordance with NIH and institutional guidelines for human subject research.

\section{Igf2bp3 siRNA transfection}

Freshly-isolated primary mouse hepatic stellate cells or human low-passage myofibroblastic stellate cells (obtained from ThermoFisher Scientific, Waltham, MA) were cultured in 10\% fetal bovine serum (FBS, Gibco, Life Technologies) and 1\% penicillin/streptomycin (P/S, Gibco, Life Technologies) at $37^{\circ} \mathrm{C}$ in a humidified atmosphere containing $5 \% \mathrm{CO}_{2}$ for 3 days. Before transfection with siRNA, the cells were serumstarved for 24 hours, and then cultured in antibiotic-free medium with $2 \%$ FBS for 24 hours. Cells were transfected with $25 \mathrm{nM}$ of human (L-003976) or mouse Igf2bp3 (L-059667) SMART-pool siRNA, a mixture of four siRNAs (Dharmacon, Lafayette CO, USA) using Lipofectamine RNAiMAX (Invitrogen, Life Technologies, Carlsbad, CA, USA) according to the manufacturer's instructions. As a negative control group, $25 \mathrm{nM}$ of nontargeting siRNA (D-001206 non- targeting siRNA, siCONTROL; Dharmacon, Lafayette CO, USA) transfected human or mouse HSCs. These cells were harvested at $24 \mathrm{~h}$ and $48 \mathrm{~h}$ after transfection, and both RNA and protein were extracted for gene expression analysis. The efficiency of gene knockdown was evaluated by qRT-PCR and Western blot assays.

\section{Quantitative real-time PCR}

Fresh cells or liver tissue that had been stored at $-80^{\circ} \mathrm{C}$ were extracted with TRIZOL ${ }^{\mathrm{TM}}(\mathrm{Ambion} 囚$ by Life Technologies) to obtain total RNA. After assuring sufficient RNA quality and concentration, gene expression was evaluated by qRT-PCR analysis. mRNAs were quantified by real-time RT-PCR using SYBR Green PCR master mix (Applied Biosystems) using a StepOnePlus ${ }^{\mathrm{TM}}$ Real-Time PCR System (Applied Biosystems). The sequences of primers to detect mouse and human genes are listed in Table I. Samples were analyzed in duplicate according to the Ct method compared to the S9 reference gene.

Western blot and ELISA assay

Total protein was extracted from freeze-clamped liver tissue samples that had been stored at $-80^{\circ} \mathrm{C}$. Whole tissues were homogenized in RIPA buffer (Thermo) supplemented with protease inhibitors. Equal 


\section{Cellular Physiology Cell Physiol Biochem 2018;48:1215-1229 \begin{tabular}{l|l|l} 
DOI: 10.1159/000491987 & $\begin{array}{l}\text { C } 2018 \text { The Author(s). Published by S. Karger AG, Basel } \\
\text { www.karger.com/cpb }\end{array}$
\end{tabular}}

Wang et al.: RNA Binding Proteins in Stellate Cell Activation

amounts of total protein were separated by polyacrylamide gel electrophoresis and transferred to PVDF (polyvinylidene difluoride) membranes. Primary antibodies against Celf2 (ab186430; Abcam), Igf2bp3 (03-198; EMD Milipore. 14642-1-AP; Proteintech Group), Rbm47 (ab167164; Abcam) and $\alpha$-SMA (ab32575; Abcam) were used, and HRPsecondary antibodies (GE). Membranes were developed by chemiluminescence (SuperSignal West, Thermo). Blots obtained from three independent experiments were scanned by ChemiDoc XRS+ (Bio-Rad), and a ROI around the band of interest was defined. Band intensities were calculated using the CS analyzer 2.0 program (ATTO Corporation).

Indirect ELISA was performed on cell lysate to quantify Hmga2 or Cdk6 from Igf2bp3 non-targeting (nt) or si-RNA (siRNA) transfected cells. For the detection of Hmga2 in cell lysate, indirect detection with alkaline phosphatase labeled anti-rabbit IgG (Sigma-Aldrich) was performed. After reacting with pNPP substrate solution (Sigma-Aldrich) quantitative analysis was determined from spectrophotometric readings at $405 \mathrm{~nm}$ (xMark, Bio-Rad, Hercules, CA).

\section{Cell Proliferation (CCK8 and BrdU) assay and Scratch assay}

Cell proliferation was measured with a Cell Counting Kit-8 Assay (Dojindo Molecular) and BrdU as described previously $[15,16]$. Briefly, cells were plated at a density of $2 \times 10^{3}$ cells per well in 96 -well plates, with the indicated treatment and time. After transfection, adding the CCK8 and BrdU reagent, the plates were incubated in a $\mathrm{CO}_{2}$ incubator at $37^{\circ} \mathrm{C}$ until the color developed. Absorbance was then measured at each wavelength of reagent using an ELISA plate reader. Standard wound healing assays were performed by growing cells to a confluent monolayer, and making a manual scratch using a $200 \mu \mathrm{L}$ pipette tip. Floating cells were washed out and fresh medium containing transfection reagent with Igf2bp3 siRNA or nontargeting siRNA was added. The width of each scratch was recorded by taking photographs $(\times 20)$ using an Olympus inverted microscope (Olympus Optical Co., Ltd.) at time 0, and 24, 36 and 48 hours after the scratch and transfection. Empty area at each time point was quantified with NIH image J software (version 1.49, NIH http://imagej.nih.gov/ij//) and compared with that in the initiation of cell migration.

\section{Pharmacologic inhibition of TGF- $\beta$ - 1 signaling in human or mouse primary HSC}

Human hepatic stellate cells were cultured in medium supplemented with $10 \%$ FBS to $70 \sim 80 \%$ confluency, and starved in $0.2 \%$ FBS-containing medium. After 24 hours, cells were incubated with $10 \mu \mathrm{M}$ SB431542 (Selleckchem, Houston, TX, USA), an inhibitor of TGF- $\beta$ R1 kinase, or with vehicle overnight (16 hours), and then total RNA was extracted from three parallel cultures. Gene expression was assessed by qRT-PCR.

Isolated mouse primary HSCs were cultured with $10 \mu \mathrm{M}$ SB431542 or $10 \mathrm{ng} / \mathrm{ml}$ TGF- $\beta$ for 7 days, or cultured for 4 days with SB431542 and then an additional 3 days with SB431542 plus TGF- $\beta$. After 7 days, cells were harvested and RNA extracted to test for gene expression by qRT-PCR.

\section{Calculation of EMT Scores}

Epithelial and mesenchymal gene expression signatures from both Tan et al. [17] and Groger et al. [18] were used in calculating the scores. Scores were computed as described in Tan et al [17]. Briefly, the signatures were applied to mouse Q-HSC and MF-HSC microarray data from Chen et al [19]. The empirical cumulative distribution function was estimated for each signature on the ranked-ordered RNA abundance of mouse Q-HSC or mouse MF-HSC cells. A two-sample Kolmogorov-Smirnov test was applied to calculate the difference between the signatures. The more positive a score the more mesenchymal-like and the more negative the more epithelial-like, with transitional cells appearing in between with a score closer to zero. 
Statistical analysis

Results are expressed as mean \pm SEM. Statistical differences were determined by Student's t-test or one-way ANOVA using SPSS Statistics 20, followed by Scheffe' post hoc test. P-values less than 0.05 were considered to be statistically significant.

\section{Results}

RBPs are differentially expressed in quiescent and myofibroblastic HSCs

To identify RBPs that changed during the myofibroblastic differentiation process, we performed microarray analysis of mouse MF-HSC and Q-HSC [19], and discovered that several RBPs were significantly induced, while others were significantly repressed (Fig. 1A, B). Because none of these RBPs were known to function in HSC, we did a literature search to determine if any of the differentially-expressed HSC RBPs had been reported to regulate fate decisions in other stem-like cells. Igf2bp3 (also known as IMP3) was the most upregulated RBP during HSC differentiation. Interestingly, we found many publications addressing IGF2BP3's significance in various stem cell populations, with evidence indicating that IGF2BP3 is considered to be an oncofetoprotein that controls epithelial-to-mesenchymal transitions (EMT) and viability in tumor initiating stem-like cells of several GI epithelial cancers, including pancreatic adenocarcinoma, cholangiocarcinoma, and hepatocellular carcinoma [20-23]. CELF2, another RBP that was significantly induced during myofibroblastic differentiation of HSC, has been strongly linked to EMT in other types of stem-like cells, including those involved in hepatocarcinogenesis [24]. Conversely, RBM47, an RBP that was profoundly suppressed in MF-HSC relative to Q-HSC, has been shown to promote mesenchymal-to-epithelial transitions (MET) in pluripotent stem cells derived from mouse embryonic fibroblasts [7, 25]. Therefore, our subsequent studies in HSC and injured livers focused on IGF2BP3, CELF2 and RBM47.

Expression of RBPS that regulate epithelial-to-mesenchymal and mesenchymal-to-epithelial transitions (EMT/MET) changes during HSC activation and fibrogenesis

qRT PCR and Western blot analyses were done to compare mRNA and protein levels of Igf $2 \mathrm{bp} 3$, Celf2 and Rbm47 in freshly isolated mouse HSC versus mouse HSC that had been cultured under standard conditions to induce their differentiation

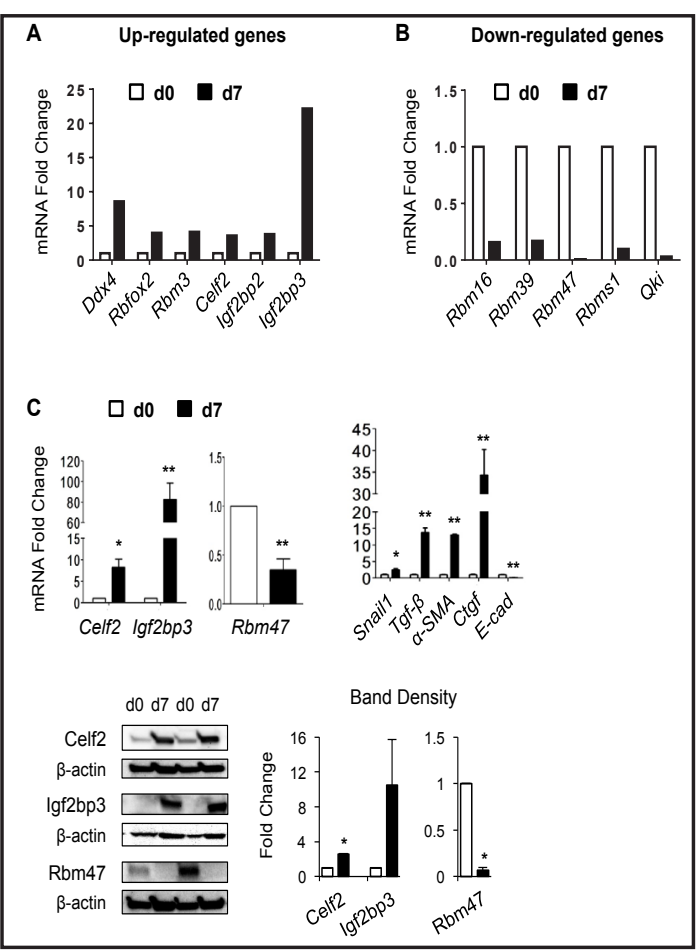

Fig. 1. RNA Binding Proteins (RBPs) are differentially expressed in quiescent and myofibroblastic murine hepatic stellate cells (HSCs). Primary HSCs were isolated from livers of normal C57BL/6 mice. Microarray analysis was performed on RNA isolated from quiescent (Q)-HSCs (d0; immediately after isolation) and myofibroblastic (MF)-HSCs (d7; cultured for 7 days to induce myofibroblastic differentiation) in triplicate [19]. Fold changes in RBPs are shown for upregulated genes (A) and down-regulated genes (B). (C) qRT-PCR for Celf2, Igf2bp3, and Rbm47, and immunoblots for Celf2, Igf2bp3, and Rbm47 in quiescent (d0) and cultureactivated HSCs (d7) isolated from mice ${ }^{* *} \mathrm{p}<0.005$ vs $\mathrm{d} 0$ ). In Western blot assays, $\beta$-actin was used as an internal control for densitometry analysis. 
into MF-HSC. In mouse HSC (Fig. 1C), expression of these genes and proteins changed as predicted by the microarray assay, demonstrating that Igf $2 \mathrm{bp} 3$ and Celf2 (the pro-EMT RBPs) were significantly up-regulated, while Rbm47 (the pro-MET/anti-EMT RBP) was significantly down-regulated, while Q-HSC activated and acquired myofibroblast markers (e.g., alpha smooth muscle actin, $\alpha$-SMA, Tgf $\beta 1$, Snai1, Ctgf) in vitro. To determine if similar reciprocal regulation of pro- and anti-EMT RBPs occurred as Q-HSC become MF-HSC in vivo, we compared mRNA expression of the respective RBPs in the livers of healthy control mice and mice with liver injury. Compared to control livers, $\mathrm{CCl}_{4}$-injured livers expressed significantly higher levels of Igf2bp3 and significantly lower levels of Rbm47 mRNA, as well as increased whole liver IGF2BP3 protein (Fig. 2A), consistent with our in vitro evidence that HSC differentiation is characterized by differential expression of these RBPs. To further investigate that possibility, we evaluated RBPs in a rat model of reversible liver fibrosis in which a fibrogenic insult (biliary obstruction due to bile duct ligation, BDL) is removed by constructing a Roux-en-Y (RY) biliary-intestinal anastomosis to decompress the obstructed biliary tree in rats. Biliary decompression reverses the chronic cholestatic liver injury and promotes regression of the cholestasis- associated liver fibrosis [26]. As predicted by our other studies, livers with BDL-related fibrosis expressed lower levels of Rbm47 than livers of sham-operated control rats, and expression of Rbm47 recovered to baseline levels of the sham-operated controls within one week of reversing the biliary obstruction in the BDL group by constructing RY anastomoses (Fig. 2B); other RBPs were not significantly affected by BDL (Fig. 2C). In addition to confirming the reciprocal relationship between fibrogenic

Fig. 2. Expression of RBPs that regulate epithelial-tomesenchymal and mesenchymalto-epithelial transitions (EMT/ MET) changes during HSC activation and fibrogenesis. (A) qRT-PCR for Igf2bp3 and Rbm47, and Western blot and cumulative densitometry analyses (relative to Gapdh control) for Igf $2 b p 3$ in livers of mice treated with $\mathrm{CCl}_{4}$ or oil vehicle (Veh) for 6 and 10 weeks. ( $\mathrm{n}=3$ mice / group) $\left({ }^{*} \mathrm{p}<0.05\right.$ vs each own control). (B) qRT- PCR for Rbm47 in livers of rats that underwent sham surgery, bile duct ligation (BDL), or BDL with subsequent Roux-en-Y biliary intestinal anastomosis (BDL-RY) ( $\mathrm{n} \geq 4$ rats / group). (C) qRT- PCR for Celf2 and Igf2bp3 in livers of rats that underwent sham surgery, bile duct ligation (BDL), or BDL with subsequent Roux-en-Y biliary intestinal anastomosis (BDL-RY)

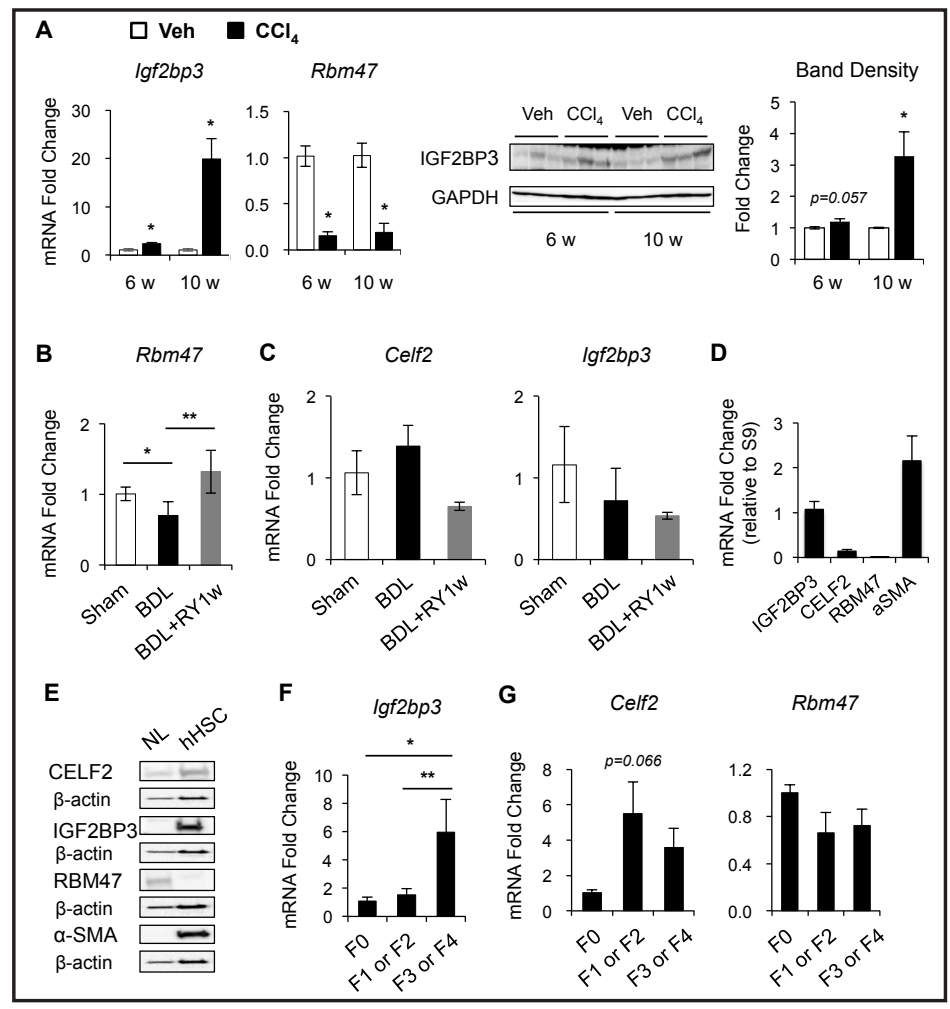
( $\mathrm{n} \geq 4$ rats / group). (D) qRT-PCR for Igf2bp3, Celf2, Rbm47 and $\alpha$-SMA, plotted as fold change relative to S9 control RNA. (E) Immunoblots for CELF2, IGF2BP3, RBM47 and $\alpha$-SMA in human pHSCs. Normal human liver (NL) is shown as a blotting control. (F) qRT-PCR for Igf2bp3 in human liver biopsies with NAFLD and different stages of liver fibrosis (F) ( $n=3-5$ patients/fibrosis stage) $\left({ }^{*} \mathrm{p}<0.05\right.$, $\left.{ }^{* *} \mathrm{p}<0.005\right)$. (G) qRT-PCR for Rbm47 and Celf2 in human liver biopsies with NAFLD at defined stages of liver fibrosis ( $\mathrm{n}=3-5$ patients/ fibrosis stage). In Western blot assays, immunoblot images shown represent one of three experiments with similar results. The mean \pm s.e.m. results obtained from at least three independent experiments are graphed. 
factors and Rbm47, these data demonstrate the dynamic nature of the RBP response and suggest that recovery of the anti-EMT/pro-MET RBP profile readily occurs even in chronically fibrotic livers.

Because the ultimate goal of our preclinical research is to identify novel therapeutic targets in humans with advanced liver fibrosis, we first tested whether myofibroblastic, lowpassage primary human stellate cells (hMF-HSC) express RBPs, and find that IGF2BP3 and CELF2 mRNAs are expressed comparably to $S 9$ control or the myofibroblast marker $\alpha$-SMA (Fig. 2D). Further, both IGF2BP3 and CELF2 are readily detected as proteins by western blot compared to protein lysates of normal human liver (NL, Fig 2E). In contrast, RBM47 RNA (the anti-EMT/pro-MET factor) is expressed at much lower levels in hMF-HSC and although RBM-47 protein is apparent on western blots of $\mathrm{NL}$, it is not detected by similar analysis of hMF-HSC lysates (Fig 2E). We then compared expression of IGF2BP3 (the RBP that was most strongly associated with the MF-HSC phenotype and liver fibrosis in our preclinical models) in liver biopsy samples from patients with nonalcoholic liver disease (NAFLD) and different stages of liver fibrosis. Compared to livers with no fibrosis (F0) or mild fibrosis (F1, F2), livers with advanced fibrosis (F3, F4) expressed significantly higher levels of IGF2BP3 (Fig. 2F). RBM47 and CELF2 did not show significant differences (Fig 2G). Therefore, the aggregate data in cultured mouse and human HSCs, in mouse and rat models of liver fibrosis, and in human liver disease identify three RBPs that are known to regulate EMT/MET in stem-like cells, as potentially novel targets to control the size of HSC-derived populations and prevent the evolution of cirrhosis during liver injury. Of these, IGF2BP3 appears the RBP that best correlates with liver injury and disease and thus, the remainder of our studies focus mainly on IGF2BP3.

\section{IGF2BP3 depletion changes HSC gene expression and biology}

To determine the role of IGF2BP3 in regulating these fate changes in HSC, we used siRNA constructs to knock down IGF2BP3 in human MF-HSC (Fig. 3A) and used RNA-seq to compare the transcriptomes of IGF2BP3-depleted MF-HSC and control MF-HSC. Depleting IGF2BP3 altered the expression of thousands of genes, and gene ontogeny/gene set enrichment analysis demonstrated that genes involved in TGF- $\beta$ signaling, regulation of cell viability and proliferation, and EMT were among the top categories of genes that were down-regulated by IGF2BP3 depletion (Fig. 3B). qRT PCR and/or Western blot analysis confirmed that depleting IGF2BP3 suppressed expression of TGF- $\beta 1$, reduced expression of TGF- $\beta$-regulated transcription factors that promote EMT (e.g., Twist1, Snai1) and decreased expression of MF-associated genes (e.g., $\alpha$-SMA, Col1 $\alpha 1$, Ctgf) but tended to increase expression of the epithelial marker, E-cadherin (Fig. 3C). To confirm that changes in gene expression had functional consequences, we assessed effects of IGF2BP3 depletion on proliferation (Fig. 3D) and migration (Fig. 3E), two key characteristics of HSC-derived MFs. Compared to MF-HSC treated with non-targeting (nt) control siRNA, IGF2BP3-depleted MF-HSC were significantly less proliferative and their migratory activity was reduced by more than $50 \%$, suggesting that IGF2BP3 typically promotes the proliferative, myofibroblastic HSC phenotype. Therefore, the aggregate data from RNA-seq, qRT PCR, and Western blot analyses complement and confirm the functional data showing that IGF2BP3 supports the myofibroblastic HSC phenotype. Interestingly, IGF2BP3-depleted HSC also expressed lower levels of CELF2 mRNA (Fig. 3A) and protein (Fig. 3C), suggesting that IGF2BP3 might promote the expression of at least one other pro-EMT RBP. In contrast, depleting IGF2BP3 did not seem to influence expression of RBM47, the RBP with putative anti-EMT/pro- MET activity (Fig. 3A, C).

Transcriptome analysis demonstrates that HSC are hybrid epithelial-mesenchymal cells

The concept that HSC transition back and forth between quiescence and their myofibroblastic phenotype by undergoing EMT/MET-like processes that are regulated by RBPs suggests that HSC may have some characteristics of transitional (also dubbed "hybrid") cells. Emerging evidence suggests that some hybrid cells, including stem-like/progenitor cells, may remain in the hybrid state indefinitely until triggered to acquire either a more 
Fig. 3. IGF2BP3 depletion changes HSC gene expression and biology. (A) qRT-PCR for Igf2bp3, Celf2 and Rbm47 in primary human HSCs transfected with non-targeting (nt) or IGF2BP3 siRNA (siRNA). (B) Heat map and Enrichment of Gene Ontology categories among genes differentially regulated in IGF2BP3 knockdown vs control human HSCs. Genes reproducibly (FDR q-value $<$ 0.05) up-regulated (upper panel) or down-regulated (lower panel) following knockdown were compared to all expressed genes for enrichments in the Biological Processes categories from the Gene Ontology Consortium database. Related pathways were grouped and color-coded. (C) qRT-PCR for TGF $\beta$, Twist, Snail1, $\alpha$-SMA, Ctgf, Collagen $1 \alpha 1(\operatorname{Col} 1 \alpha 1)$ and E-cadherin (E-cad) and cumulative densitometry analyses for IGF2BP3, CELF2, RBM47 and $\alpha$-SMA in human pHSCs transfected with nt or siRNA. (D) Cell proliferative activity was quantified by CCK8 and BrdU assay

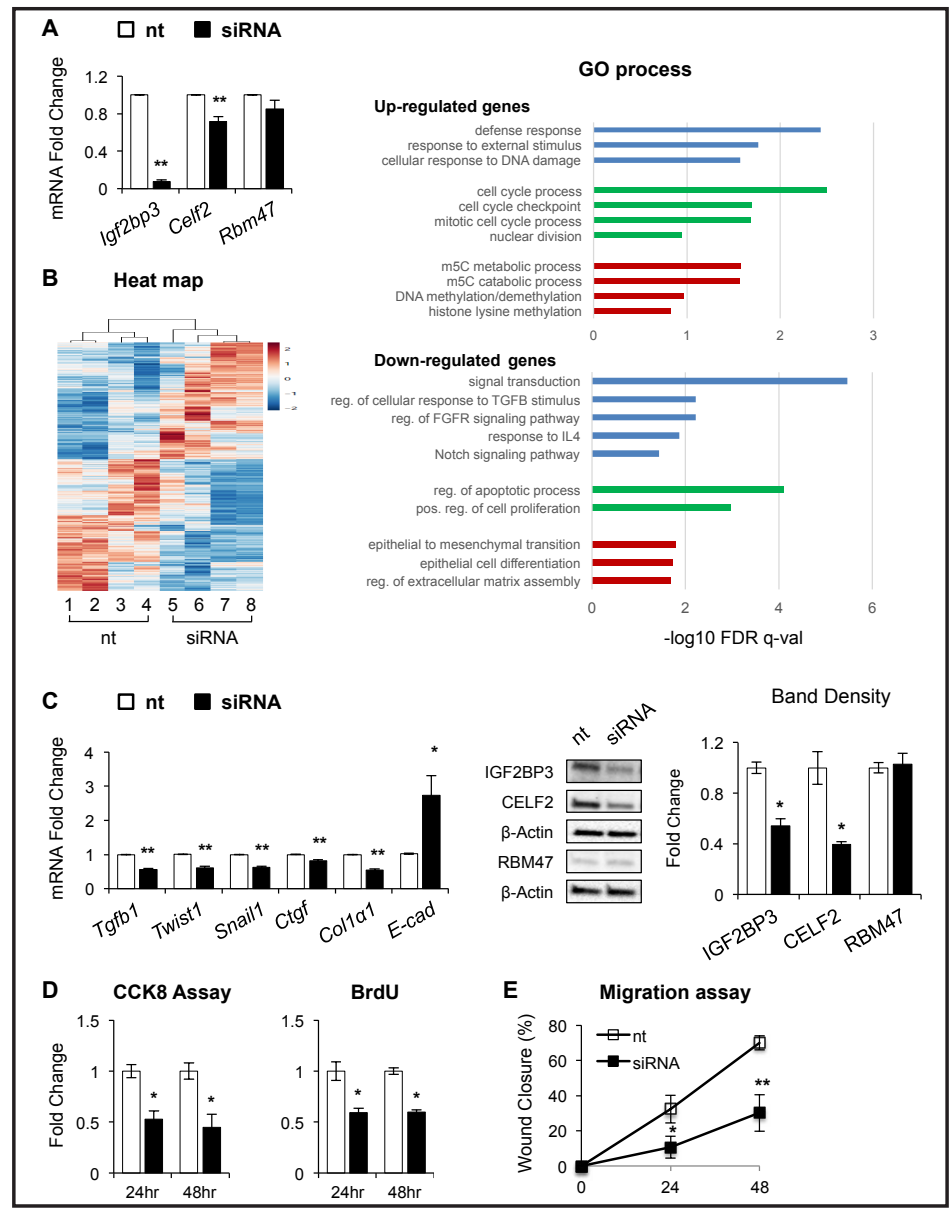
in these cells. (E) Cell migration

was assessed by wound healing assays in human pHSCs at 0, 24 and 48 hours after transfection with nt or siRNA. The mean \pm s.e.m. results obtained from at least three independent experiments are graphed $\left({ }^{*} \mathrm{p}<0.05,{ }^{* *} \mathrm{p}<0.005\right.$ vs nt).

completely mesenchymal or epithelial fate $[27,28]$ HSC have mesenchymal stem cell-like attributes [8, 29-31] and reports indicate that HSC co-express epithelial and mesenchymal markers in certain contexts [5, 29, 30, 32, 33]. We evaluated the transcriptomes of Q-HSC and MF-HSC using genomic signatures generated from multiple meta-analyses in malignancy and embryogenesis, states that are known to be controlled by EMT/MET in stem-like cells. Wellaccepted algorithms applied to these signatures can distinguish epithelial or mesenchymal cells from transitional/hybrid cells $[17,18]$. When applied to our mouse HSC RNA sequencing data [19], these algorithms indicate that primary HSCs fulfill gene expression criteria for transitional cells, i.e., they co- express numerous epithelial and mesenchymal genes (Fig. 4A, B). We also observed that HSC cells acquire a more mesenchymal-like expression signature during activation of the myofibroblastic phenotype. Importantly, co-expression of certain epithelial genes is maintained even after HSCs acquire a myofibroblastic phenotype, consistent with reports that MF-HSC retain sufficient plasticity to undergo reprogramming back to a more quiescent and less mesenchymal state $[9,10,32]$.

IGF2BP3 physically interacts with HmgA2 mRNA to stabilize expression of this master regulator of epithelial-mesenchymal transitions during HSC differentiation

In other cell types Igf $2 \mathrm{bp} 3$ promotes EMT, in part, by directly binding to and stabilizing mRNA encoding Hmga2, a master regulator of other pro-EMT transcription factors, including Twist1 and Snai1 [28]. We found that Twist1 and Snai1 were sensitive to Igf2bp3 depletion 
in MF-HSC (Fig. 3C). Therefore, we used several complementary approaches to evaluate the possibility that Igf2bp3-Hmga2 interaction may be involved in Igf2bp3's pro-EMT activity in HSC. Microarray analysis of primary mouse HSCs demonstrated that like Igf2bp3 (Fig. 1A), Hmga2 expression increased significantly during myofibroblastic differentiation of HSC (Fig. 5A). RNA immunoprecipitation (RIP) of IGF2BP3 in human MF-HSCs confirmed direct physical interaction between IGF2BP3 protein and Hmga2 mRNA (Fig. 5B). Further, separate experiments that used siRNA to deplete IGF2BP3 in MF-HSC demonstrated that net mRNA expression of Hmga2 fell significantly when IGF2BP3 expression was reduced (Fig. 5C). Parallel decreases in HMGA2 protein were confirmed by ELISA (Fig 5D). Together, these data support the concept that IGF2BP3 directly interacts with and stabilizes Hmga2 mRNA to promote EMT in HSC, as has been reported to occur in other cell types [23].

IGF2BP3 promotes MF-HSC growth and proliferation by stabilizing the insulin like growth factor pathway and cyclin-dependent kinase 6

Myofibroblasts typically thrive and proliferate in TGF- $\beta$-enriched microenvironments, and our RNA-seq/Gene Ontogeny analyses indicated that IGF2BP3 depletion reduces TGF- $\beta$ signaling and inhibits cell growth and proliferative activity (Fig. 3). In other cell types, TGF- $\beta$ induces IGF2BP3 $[21,34]$ and IGF2BP3 enhances cell growth by enhancing insulin-

Fig. 4. Quiescent murine HSCs contain global gene expression signatures similar to epithelial-tomesenchymal transitional cells and become more mesenchymal after activation. (A) Cumulative distribution function plots depict the fraction of epithelial signatures (red) or mesenchymal signatures (blue) as a function of absolute ranked RNA abundance, using the epithelial and mesenchymal signatures from the meta-analysis by Tan et al [17]. The further the signature is to the right of the plot indicates that a higher proportion of the genes in the signature are among the most abundantly expressed genes in the sample. EMT scores are on a scale of -1 to +1 , with positive scores indicating more mesenchymal, negative scores more epithelial, and transitional cells closer to zero. (B) The same analysis using the epithelial and mesenchymal signatures

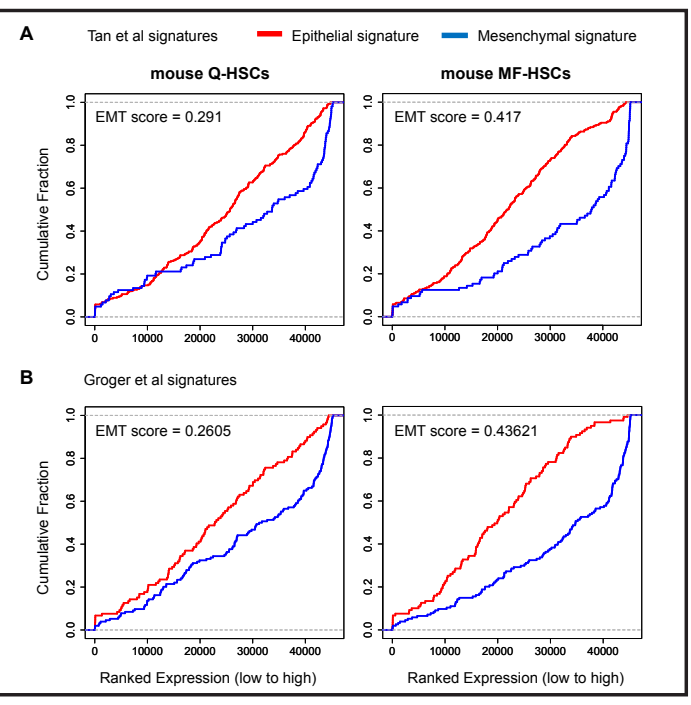
from the meta-analysis by Groger et al [18].

Fig. 5. IGF2BP3 physically interacts with Hmga2 mRNA to stabilize expression of this master regulator of epithelialmesenchymal transitions during HSC transdifferentiation. (A) Microarray analysis for Hmga2 in mouse pHSCs ${ }^{* *} \mathrm{p}<0.005$ vs d0). (B) RNA immunoprecipitation of IGF2BP3 in human pHSCs. Bar graphs comparing the fold-enrichment for Hmga2, Rps5, and Gapdh in control (rabbit IgG) versus anti-IGF2BP3 antibody RNA immunoprecipitation (RIP). Levels of these genes are normalized to input levels from total RNA with RPL8 (ribosomal protein, large subunit 8). Two mRNAs that are not targets of IGF2BP3 (GAPDH and RPS5) are not enriched. (C) qRT-PCR and (D) ELISA for Hmga2 in human pHSCs transfected with non-targeting (nt) or IGF2BP3 siRNA (siRNA) $\left({ }^{*} \mathrm{p}<0.05\right.$ vs $n t)$. The mean \pm s.e.m. results obtained from at least three independent experiments are graphed.

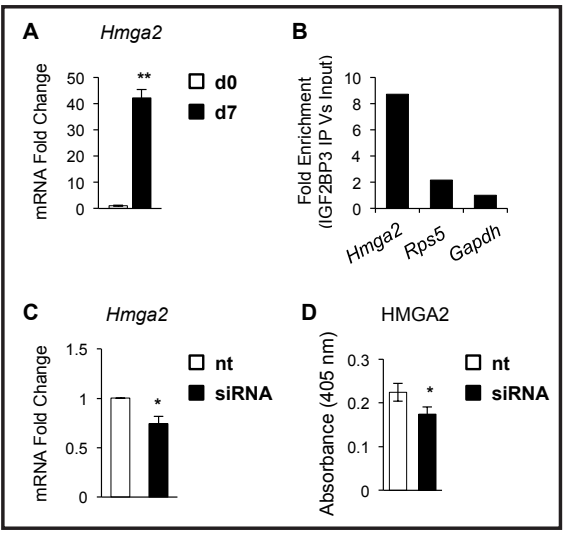


like growth factor (IGF) signaling. More specifically, IGF2BP3 stabilizes expression of IGF2, its receptor IGF1R, and another IGF2 binding protein, IGF2BP1. IGF1R signaling ultimately results in activation of PI3K and its target, AKT $[35,36]$. Our HSC microarray analysis confirmed that expression of Igf1R and Igf $2 \mathrm{bp} 1$ both increase in parallel with Igf2bp3 as mouse Q-HSC differentiate into MF (Fig. 6A), and HSC growth and viability are known to depend upon PI3K/AKT activity [37]. Therefore, Igf2bp3-mediated enhancement of IGF1R signaling is predicted to promote the growth of MF-HSC and likely explains why we observed that IGF2BP3 depletion inhibited MF-HSC growth in culture (Fig. 3D). IGF2BP3 is also known to directly bind to and stabilize expression of CDK6 [38-40], a kinase that interacts with cyclin D3 to promote proliferation in hepatocellular- and cholangio-carcinomas [41]. We found that depleting IGF2BP3 reduced the proliferative activity of MF-HSC, as evidenced by decreased incorporation of BrdU (Fig. 3D). Hence, we used RIP to determine if IGF2BP3 directly interacts with and stabilized Cdk6 mRNA in MF-HSCs. IGF2BP3 immunoprecipitates were significantly enriched with Cdk6 mRNA (Fig. 6B) and knocking down IGF2BP3 significantly decreased steady state levels of Cdk6 mRNA (Fig. 6C) and protein content (Fig 6D), showing that IGF2BP3 stabilizes Cdk6 in HSC and suggesting an additional mechanism for the trophic actions of IGF2BP3 in MF-HSC.

\section{Igf2 $\mathrm{bp} 3$ is both a target and effector of TGF- $\beta$ signaling in HSCs}

Having shown that Igf2bp3 plays significant roles in promoting the myofibroblastic fate of HSC, we wished to identify mechanisms that regulate HSC expression of IGF2BP3. In other cell types IGF2BP3 induction is a downstream consequence of signaling initiated by TGF- $\beta$ and involves TGF- $\beta$-mediated down-regulation of Let7 microRNAs that typically suppress expression of Igf2 bp3 mRNA [42]. We profiled microRNAs in primary mouse HSC at various points during the process of culture-induced myofibroblastic differentiation and discovered that freshly isolated Q-HSC robustly express Let7i. Further, we found that expression of Let7i progressively declines to a fraction of the basal levels by culture day 7 (Fig. 7A) when the stellate cells are fully myofibroblastic and express 80 -fold higher levels of Igf $2 \mathrm{bp} 3 \mathrm{mRNA}$ (Fig. 1C). qRT PCR analysis of RNA from freshly-isolated and culture-activated mouse HSC confirmed expected increases in the expression of Tgf- $\beta 1$ and Tgf- $\beta$ receptor- 1 (Tgf$\beta R 1$ ) in MF-HSC relative to Q-HSC (Fig. 7B), consistent with many reports demonstrating that production of TGF- $\beta 1$ by MF-HSC functions in an autocrine fashion to maintain their myofibroblastic phenotype (reviewed in $[1,4]$ ). In accordance with this concept, the typical culture-related induction of several myofibroblast-associated genes, including Igf2bp3, $\alpha$-SMA and Col1 $\alpha 1$, was significantly attenuated in HSC that were cultured with the Tgf- $\beta$ receptor-1 inhibitor, SB431542. Conversely, adding Tgf- $\beta 1$ to HSC cultures further enhanced induction of Igf $2 \mathrm{bp} 3$ and the other myofibroblast markers, and these effects were blocked by SB431542 (Fig. 7C). Importantly, treating low passage hMF-HSC with this TGF- $\beta$ R1

Fig. 6. IGF2BP3 promotes MF-HSC growth and proliferation by stabilizing the insulin like growth factor pathway and cyclindependent kinase (Cdk6), and its expression is regulated by TGF- $\beta$. (A) Microarray data for Igf1r and Igf2bp1 in mouse pHSCs, day 0 vs day 7 ( $^{* *} \mathrm{p}<0.005 \mathrm{~d} 7 \mathrm{vs} \mathrm{d} 0$ ). (B) RNA immunoprecipitation of IGF2BP3 in human pHSCs. Bar graphs comparing the foldenrichment for Cdk6, Rps5, and Gapdh in control (rabbit IgG) and anti-IGF2BP3 antibody RNA immunoprecipitation (RIP). Levels of these genes are normalized to input levels from total RNA with RPL8 (ribosomal protein, large subunit 8). Two mRNAs that are not targets of IGF2BP3 (Gapdh and Rps5) are not enriched. (C) qRT- PCR and (D) ELISA for Cdk6 in human pHSCs transfected with non-targeting (nt) or IGF2BP3 siRNA

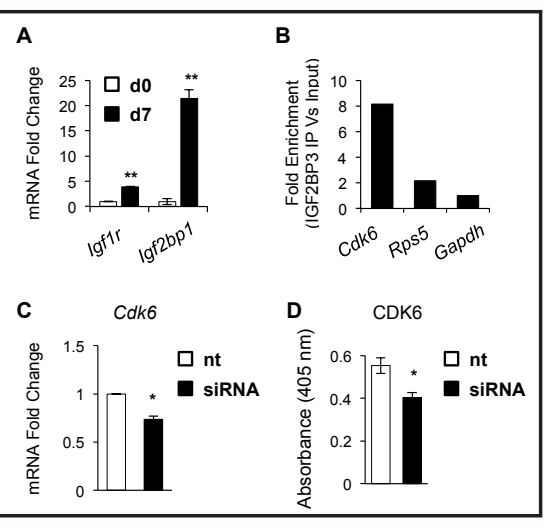
(siRNA). The mean \pm s.e.m. results obtained from at least three independent experiments are graphed. ( $\left.{ }^{*} \mathrm{p}<0.05 \mathrm{vs} n t\right)$. 
Fig. 7. IGF2BP3 is both a target and effector of TGF- $\beta$ signaling in HSCs. (A) qPCR analysis of let7i miRNA in rat $\mathrm{pHSC}$ on culture day (d) 0, 1, 4 and 7. (B) TGF- $\beta 1$ and TGF- $\beta$ R1 expression in $\mathrm{Q}$ and $\mathrm{MF}$ murine pHSC by qRT-PCR analysis. Data are graphed as mean \pm SEM for 3 independent experiments. * $\mathrm{p}<0.05$ vs d0. (C) Inhibiting TGF$\beta R 1$ activity with SB431542 (ALK5 inhibitor) reduces expression of Igf $2 \mathrm{bp} 3, \alpha$-SMA and Col1 $\alpha 1$ myofibroblast markers in primary mouse HSC. Data are graphed as mean +/- SEM for 3 independent experiments. ${ }^{*} \mathrm{p}<0.05$ vs $\mathrm{d} 7$. (D) Inhibiting TGF- $\beta R 1$ activity with SB431542 (ALK5 inhibitor) differentially regulates expression of Let7i, and inhibits Igf2bp3 and $\alpha$-SMA in human pHSC. Data are graphed as mean +/- SEM for 3 independent experiments. * $\mathrm{p}<0.05$ vs vehicle. (E) Gene Set Enrichment Analysis (GSEA) of RNA-seq data from human pHSC treated with siRNA for Igf2bp3.

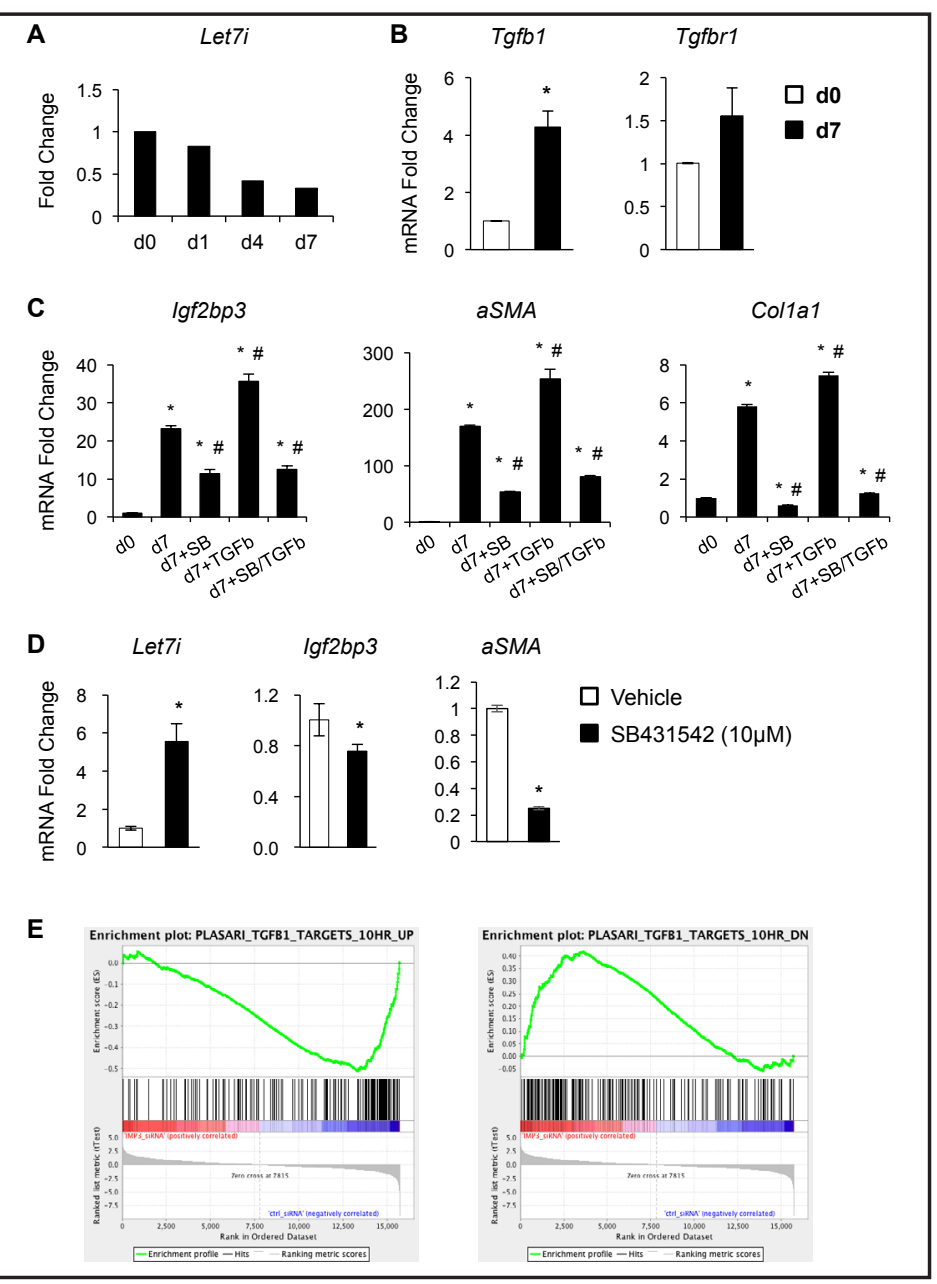

-inhibitor (which is known to suppress the myofibroblastic phenotype in HSC [43] and which reduced $\alpha$-SMA expression in our hMF-HSCs by $\sim 75 \%$ ), increased Let7i and coincidentally suppressed accumulation of Igf $2 \mathrm{bp} 3$ mRNA (Fig. 7D). These results show that Igf2bp3 is a target of TGF- $\beta$ signaling in HSCs and suggest that Let7 miRs are the proximal mediators of TGF- $\beta$-dependent induction of Igf $2 \mathrm{bp} 3$ in these cells, as has been noted in other cell types [42]. Importantly, gene enrichment set analysis of RNA-seq data from human pHSCs that were treated with IGF2BP3 siRNA demonstrate that depletion of IGF2BP3 itself abrogates the effects of TGF- $\beta$ signaling on multiple other TGF- $\beta$ targets that promote typical traits of MF-HSC (Fig. 7E). The latter include TGF- $\beta$-induced genes that enhance HSC proliferative and migratory capabilities, two key functions of MF-HSC that were inhibited by knocking down Igf2bp3 (Fig. 3D, E).

\section{Discussion}

Our study is the first to show that TGF- $\beta$, a master regulator of liver fibrosis, drives myofibroblastic differentiation of HSCs by regulating microRNAs (e.g., Let7) that target RNA binding proteins (e.g., Igf2 bp3), which orchestrate coordinated changes in the expression of thousands of genes so that HSC can escape quiescence to become (and remain) proliferative myofibroblasts (Fig. 7). These findings have exciting therapeutic implications for cirrhosis because cirrhosis results from the excessive and persistent accumulation of HSC-derived myofibroblasts (MF-HSC), regardless of the primary cause of liver injury [2]. Our RNA- 
seq data show that Igf2bp3 is a distal, but critical, downstream effector of TGF- $\beta$ signaling because knocking it down is sufficient to abrogate the effects of TGF- $\beta$ on multiple processes that enable HSC to acquire and retain a proliferative, myofibroblastic state in culture. Consequently, migratory capacity, proliferative activity, fibrogenesis, and growth were all significantly inhibited in Igf2bp3-depleted HSC, and the cells reverted to a more quiescent phenotype despite persistent exposure to culture conditions that promoted myofibroblastic traits in wild type HSC. We also found that hepatic expression of Igf $2 \mathrm{bp} 3$ increases in parallel with MF accumulation and fibrosis severity in both mouse and rat models of liver fibrosis, and in patients with nonalcoholic fatty liver disease. In aggregate, these discoveries justify further research to determine whether preventing Igf2bp3 accumulation will also prevent MF accumulation and block fibrosis progression in injured livers. This possibility is highly credible based on evidence that simply blocking Igf $2 \mathrm{bp} 3$ accumulation in intact mice was sufficient to prevent hepatocarcinogenesis [21, 42], another complex pathologic process that requires Ifg2bp3-expressing stem-like cells [44]. Evidence that reducing Igf2bp3 might inhibit both liver fibrosis and carcinogenesis is particularly exciting given that cirrhosis is the major risk factor for primary liver cancer [45].

Consensus about the importance of Igf $2 \mathrm{bp} 3$ as a conserved regulator of cell plasticity is emerging $[46,47]$. However, Igf2bp3 is merely one of several RBPs that have been shown to regulate epithelial-to- mesenchymal transitions (EMT) and mesenchymal-to-epithelial transitions (MET) during development and carcinogenesis. Indeed, we discovered that Celf2 and Rbm47, two other RBPs that have been reported to reciprocally regulate EMT/MET in stem-like cells $[7,24]$, are also differentially regulated as HSC become MF. Although relatively little is known about the RBP interactome that controls these complex fate decisions in any cell type, we found that knocking down Igf $2 \mathrm{bp} 3$ also suppressed accumulation of Celf2 in HSC. Thus, we propose that these two RBPs may be components of a larger RNA regulon that orchestrates EMT. Defining this regulon will be important because the capacity for EMT/MET is a defining characteristic of hybrid cells (i.e., highly plastic cells that typically co-express epithelial and mesenchymal genes) [28], and many stem/progenitor-like cells are hybrid cells [48]. Our analyses of HSC microarray and RNA-seq data confirmed that HSC fulfill accepted criteria for "hybrid" cells, supporting evidence that HSC are liver-resident members of a larger mesenchymal stem cell-like pericyte population. It is generally accepted that these tissue-resident pericyte populations give rise to the bulk of the fibrogenic myofibroblasts that drive organ fibrosis. Hence, the concept that RNA regulons might be targeted in such cells to reduce their content of multiple different proteins that, together, support an undesirable, myofibroblastic phenotype is novel for the fibrogenesis field and has implications that extend beyond cirrhosis per se. This approach might be particularly attractive from a therapeutic risk/benefit perspective in adults with chronic injury in some, but not other, tissues because our liver data suggest that assembly of the putative Igf2bp3related EMT regulon is significantly induced by injury but negligible in healthy tissue.

\section{Abbreviations}

MF (Myofibroblasts); HSC (Hepatic stellate cells); RBPs (RNA binding proteins); Igf2bp3 (Insulin like growth factor 2 binding protein 3); CELF2 (CUGBP Elav-Like Family Member 2); RBM47 (RNA Binding Motif Protein 47); EMT (Epithelial-to-mesenchymal transitions); MET (Mesenchymal-to-epithelial transitions ); TGF- $\beta$ (Transforming growth factor beta); BDL (Bile duct ligation); NAFLD (Nonalcoholic liver disease); $\alpha$-SMA (Alpha smooth muscle actin); Col1 $\alpha 1$ (Collagen 1 alpha 1); Ctgf (Connective tissue growth factor); RIP (RNA immunoprecipitation).

\section{Acknowledgements}

The human liver specimens for this study were provided by the Duke University School of Medicine Tissue Bank Shared Resource. All samples derived from the Duke University Medical Center were obtained with informed consent under institutional review board- 


\section{Cellular Physiology Cell Physiol Biochem 2018;48:1215-1229 \begin{tabular}{l|l} 
and Biochemistry Published online: July 25, 2018 & $\begin{array}{l}\text { C } 2018 \text { The Author(s). Published by S. Karger AG, Basel } \\
\text { www.karger.com/cpb }\end{array}$
\end{tabular}}

Wang et al.: RNA Binding Proteins in Stellate Cell Activation

approved protocol Pro00005368. This work was supported by NIH grants DK106633, AA010154 and DK077794, a grant from the Duke University School of Medicine, and the Florence McAlister Professorship of Medicine, to AMD; and by the National Research Foundation (NRF) of Korea (2016R1A2B2007922) to YJ.

Conceived and planned the study (YJ, JKeene, AMD), performed experiments (SW, YJ, JH, MF, SHO, JKim, RTP), analyzed and interpreted data (YJ, MF, RTP, JKeene, AMD), wrote the manuscript (AMD, YJ), edited the manuscript (JH, MF, RTP, JKeene), obtained research funding (AMD).

\section{Disclosure Statement}

The authors declare they have no conflict of interest.

\section{References}

1 Higashi T, Friedman SL, Hoshida Y: Hepatic stellate cells as key target in liver fibrosis. Adv Drug Deliv Rev 2017;121:27-42.

- Mederacke I, Hsu CC, Troeger JS, Huebener P, Mu X, Dapito DH, Pradere JP, Schwabe RF: Fate tracing reveals hepatic stellate cells as dominant contributors to liver fibrosis independent of its aetiology. Nat Commun 2013;4:2823.

-3 Tsuchida T, Friedman SL: Mechanisms of hepatic stellate cell activation. Nat Rev Gastroenterol Hepatol 2017;14:397-411.

-4 Friedman SL: Hepatic stellate cells: protean, multifunctional, and enigmatic cells of the liver. Physiol Rev 2008;88:125-172.

-5 Michelotti GA, Xie G, Swiderska M, Choi SS, Karaca G, Kruger L, Premont R, Yang L, Syn WK, Metzger D, Diehl AM: Smoothened is a master regulator of adult liver repair. J Clin Invest 2013;123:2380-2394. Keene JD: RNA regulons: coordination of post-transcriptional events. Nat Rev Genet 2007;8:533-543. Cieply B, Park JW, Nakauka-Ddamba A, Bebee TW, Guo Y, Shang X, Lengner CJ, Xing Y, Carstens RP: Multiphasic and Dynamic Changes in Alternative Splicing during Induction of Pluripotency Are Coordinated by Numerous RNA-Binding Proteins. Cell Rep 2016;15:247-255.

8 Kramann R, Schneider RK, DiRocco DP, Machado F, Fleig S, Bondzie PA, Henderson JM, Ebert BL, Humphreys BD: Perivascular Gli1+ progenitors are key contributors to injury-induced organ fibrosis. Cell Stem Cell 2015;16:51-66.

-9 Kisseleva T, Cong M, Paik Y, Scholten D, Jiang C, Benner C, Iwaisako K, Moore-Morris T, Scott B, Tsukamoto H, Evans SM, Dillmann W, Glass CK, Brenner DA: Myofibroblasts revert to an inactive phenotype during regression of liver fibrosis. Proc Natl Acad Sci U S A 2012;109:9448-9453.

$>10$ Troeger JS, Mederacke I, Gwak GY, Dapito DH, Mu X, Hsu CC, Pradere JP, Friedman RA, Schwabe RF: Deactivation of hepatic stellate cells during liver fibrosis resolution in mice. Gastroenterology 2012;143:1073-1083 e1022.

11 Puche JE, Saiman Y, Friedman SL: Hepatic stellate cells and liver fibrosis. Compr Physiol 2013;3:1473-1492.

-12 Xie G, Swiderska-Syn M, Jewell ML, Machado MV, Michelotti GA, Premont RT, Diehl AM: Loss of pericyte smoothened activity in mice with genetic deficiency of leptin. BMC Cell Biol 2017;18:20.

$\checkmark 13$ Omenetti A, Porrello A, Jung Y, Yang L, Popov Y, Choi SS, Witek RP, Alpini G, Venter J, Vandongen HM, Syn WK, Baroni GS, Benedetti A, Schuppan D, Diehl AM: Hedgehog signaling regulates epithelial-mesenchymal transition during biliary fibrosis in rodents and humans. J Clin Invest 2008;118:3331-3342.

14 Hyun J, Wang S, Kim J, Rao KM, Park SY, Chung I, Ha CS, Kim SW, Yun YH, Jung Y: MicroRNA-378 limits activation of hepatic stellate cells and liver fibrosis by suppressing Gli3 expression. Nat Commun 2016;7:10993.

15 Syn WK, Witek RP, Curbishley SM, Jung Y, Choi SS, Enrich B, Omenetti A, Agboola KM, Fearing CM, Tilg H, Adams DH, Diehl AM: Role for hedgehog pathway in regulating growth and function of invariant NKT cells. Eur J Immunol 2009;39:1879-1892.

16 Machado MV, Michelotti GA, Jewell ML, Pereira TA, Xie G, Premont RT, Diehl AM: Caspase-2 promotes 


\section{Cellular Physiology Cell Physiol Biochem 2018;48:1215-1229 \begin{tabular}{l|l} 
and Biochemistry Published online: July 25, 2018 & $\begin{array}{l}\text { (c) } 2018 \text { The Author(s). Published by S. Karger AG, Basel } \\
\text { www.karger.com/cpb }\end{array}$
\end{tabular}}

obesity, the metabolic syndrome and nonalcoholic fatty liver disease. Cell Death Dis 2016;7:e2096.

17 Tan TZ, Miow QH, Miki Y, Noda T, Mori S, Huang RY, Thiery JP: Epithelial-mesenchymal transition spectrum quantification and its efficacy in deciphering survival and drug responses of cancer patients. EMBO Mol Med 2014;6:1279-1293.

18 Groger CJ, Grubinger M, Waldhor T, Vierlinger K, Mikulits W: Meta-analysis of gene expression signatures defining the epithelial to mesenchymal transition during cancer progression. PLoS One 2012;7:e51136.

19 Chen Y, Choi SS, Michelotti GA, Chan IS, Swiderska-Syn M, Karaca GF, Xie G, Moylan CA, Garibaldi F, Premont R, Suliman HB, Piantadosi CA, Diehl AM: Hedgehog controls hepatic stellate cell fate by regulating metabolism. Gastroenterology 2012;143:1319-1329 e1311-1311.

20 Nischalke HD, Schmitz V, Luda C, Aldenhoff K, Berger C, Feldmann G, Sauerbruch T, Spengler U, Nattermann J: Detection of IGF2BP3, HOXB7, and NEK2 mRNA expression in brush cytology specimens as a new diagnostic tool in patients with biliary strictures. PLoS One 2012;7:e42141.

-21 Nguyen LH, Robinton DA, Seligson MT, Wu L, Li L, Rakheja D, Comerford SA, Ramezani S, Sun X, Parikh MS, Yang EH, Powers JT, Shinoda G, Shah SP, Hammer RE, Daley GQ, Zhu H: Lin28b is sufficient to drive liver cancer and necessary for its maintenance in murine models. Cancer Cell 2014;26:248-261.

-22 Li M, Zhang L, Ge C, Chen L, Fang T, Li H, Tian H, Liu J, Chen T, Jiang G, Xie H, Cui Y, Yao M, Li J: An isocorydine derivative (d-ICD) inhibits drug resistance by downregulating IGF2BP3 expression in hepatocellular carcinoma. Oncotarget 2015;6:25149-25160.

-23 Ennajdaoui H, Howard JM, Sterne-Weiler T, Jahanbani F, Coyne DJ, Uren PJ, Dargyte M, Katzman S, Draper JM, Wallace A, Cazarez O, Burns SC, Qiao M, Hinck L, Smith AD, Toloue MM, Blencowe BJ, Penalva LO, Sanford JR: IGF2BP3 Modulates the Interaction of Invasion-Associated Transcripts with RISC. Cell Rep 2016;15:1876-1883.

24 Zhang L, Liu X, Zhang X, Chen R: Identification of important long non-coding RNAs and highly recurrent aberrant alternative splicing events in hepatocellular carcinoma through integrative analysis of multiple RNA-Seq datasets. Mol Genet Genomics 2016;291:1035-1051.

25 Yeganeh M, Seyedjafari E, Kamrani FA, Ghaemi N: RNA-binding protein Rbm47 binds to Nanog in mouse embryonic stem cells. Mol Biol Rep 2013;40:4391-4396.

26 Omenetti A, Popov Y, Jung Y, Choi SS, Witek RP, Yang L, Brown KD, Schuppan D, Diehl AM: The hedgehog pathway regulates remodelling responses to biliary obstruction in rats. Gut 2008;57:1275- 1282.

27 Jolly MK, Tripathi SC, Jia D, Mooney SM, Celiktas M, Hanash SM, Mani SA, Pienta KJ, Ben- Jacob E, Levine H: Stability of the hybrid epithelial/mesenchymal phenotype. Oncotarget 2016;7:27067- 27084.

28 Nieto MA, Huang RY, Jackson RA, Thiery JP: Emt: 2016Cell 2016;166:21-45.

29 Kordes C, Sawitza I, Muller-Marbach A, Ale-Agha N, Keitel V, Klonowski-Stumpe H, Haussinger D: CD133+ hepatic stellate cells are progenitor cells. Biochem Biophys Res Commun 2007;352:410-417.

-30 Kordes C, Sawitza I, Haussinger D: Hepatic and pancreatic stellate cells in focus. Biol Chem 2009;390:10031012.

31 Hindley CJ, Mastrogiovanni G, Huch M: The plastic liver: differentiated cells, stem cells, every cell? J Clin Invest 2014;124:5099-5102.

-32 Sicklick JK, Choi SS, Bustamante M, McCall SJ, Perez EH, Huang J, Li YX, Rojkind M, Diehl AM: Evidence for epithelial-mesenchymal transitions in adult liver cells. Am J Physiol Gastrointest Liver Physiol 2006;291:G575-583.

-33 Choi SS, Syn WK, Karaca GF, Omenetti A, Moylan CA, Witek RP, Agboola KM, Jung Y, Michelotti GA, Diehl AM: Leptin promotes the myofibroblastic phenotype in hepatic stellate cells by activating the hedgehog pathway. J Biol Chem 2010;285:36551-36560.

-34 Park JT, Kato M, Lanting L, Castro N, Nam BY, Wang M, Kang SW, Natarajan R: Repression of let-7 by transforming growth factor-beta1-induced Lin28 upregulates collagen expression in glomerular mesangial cells under diabetic conditions. Am J Physiol Renal Physiol 2014;307:F1390-1403.

35 Suvasini R, Shruti B, Thota B, Shinde SV, Friedmann-Morvinski D, Nawaz Z, Prasanna KV, Thennarasu K, Hegde AS, Arivazhagan A, Chandramouli BA, Santosh V, Somasundaram K: Insulin growth factor-2 binding protein 3 (IGF2BP3) is a glioblastoma-specific marker that activates phosphatidylinositol 3-kinase/mitogen-activated protein kinase (PI3K/MAPK) pathways by modulating IGF-2. J Biol Chem 2011;286:25882-25890. 


\section{Cellular Physiology Cell Physiol Biochem 2018;48:1215-1229 \begin{tabular}{l|l} 
DOI: 10.1159/000491987 & $\begin{array}{l}\text { O 2018 The Author(s). Published by S. Karger AG, Basel } \\
\text { www.karger.com/cpb }\end{array}$
\end{tabular} \\ Wang et al.: RNA Binding Proteins in Stellate Cell Activation}

-36 Hartmann EM, Bea S, Navarro A, Trapp V, Campo E, Ott G, Rosenwald A: Increased tumor cell proliferation in mantle cell lymphoma is associated with elevated insulin-like growth factor 2 mRNA- binding protein 3 expression. Mod Pathol 2012;25:1227-1235.

37 Yang L, Wang Y, Mao H, Fleig S, Omenetti A, Brown KD, Sicklick JK, Li YX, Diehl AM: Sonic hedgehog is an autocrine viability factor for myofibroblastic hepatic stellate cells. J Hepatol 2008;48:98-106.

-38 Zhu H, Wang G, Zhou X, Song X, Gao H, Ma C, Chang H, Li H, Liu FF, Lu J, Ma J: miR-1299 suppresses cell proliferation of hepatocellular carcinoma (HCC) by targeting CDK6. Biomed Pharmacother 2016;83:792797.

-39 Sanchez DI, Gonzalez-Fernandez B, San-Miguel B, de Urbina JO, Crespo I, Gonzalez-Gallego J, Tunon MJ: Melatonin prevents deregulation of the sphingosine kinase/sphingosine 1-phosphate signaling pathway in a mouse model of diethylnitrosamine-induced hepatocellular carcinoma. J Pineal Res 2017;62

40 Palanichamy JK, Tran TM, Howard JM, Contreras JR, Fernando TR, Sterne-Weiler T, Katzman S, Toloue M, Yan W, Basso G, Pigazzi M, Sanford JR, Rao DS: RNA-binding protein IGF2BP3 targeting of oncogenic transcripts promotes hematopoietic progenitor proliferation. J Clin Invest 2016;126:1495- 1511.

41 Huang CK, Aihara A, Iwagami Y, Yu T, Carlson R, Koga H, Kim M, Zou J, Casulli S, Wands JR: Expression of transforming growth factor beta1 promotes cholangiocarcinoma development and progression. Cancer Lett 2016;380:153-162.

42 Fawzy IO, Hamza MT, Hosny KA, Esmat G, Abdelaziz AI: Abrogating the interplay between IGF2BP1, 2 and 3 and IGF1R by let-7i arrests hepatocellular carcinoma growth. Growth Factors 2016;34:42-50.

43 Zhao C, Chen W, Yang L, Chen L, Stimpson SA, Diehl AM: PPARgamma agonists prevent TGFbeta1/Smad3signaling in human hepatic stellate cells. Biochem Biophys Res Commun 2006;350:385-391.

44 Machida K, Chen CL, Liu JC, Kashiwabara C, Feldman D, French SW, Sher L, Hyeongnam JJ, Tsukamoto H: Cancer stem cells generated by alcohol, diabetes, and hepatitis C virus. J Gastroenterol Hepatol 2012;27 Suppl 2:19-22.

45 Pinter M, Trauner M, Peck-Radosavljevic M, Sieghart W: Cancer and liver cirrhosis: implications on prognosis and management. ESMO Open 2016;1:e000042.

46 Mueller-Pillasch F, Pohl B, Wilda M, Lacher U, Beil M, Wallrapp C, Hameister H, Knochel W, Adler G, Gress TM: Expression of the highly conserved RNA binding protein KOC in embryogenesis. Mech Dev 1999;88:95-99.

-47 Bell JL, Wachter K, Muhleck B, Pazaitis N, Kohn M, Lederer M, Huttelmaier S: Insulin-like growth factor 2 mRNA-binding proteins (IGF2BPs): post-transcriptional drivers of cancer progression? Cell Mol Life Sci 2013;70:2657-2675.

48 Jolly MK, Jia D, Boareto M, Mani SA, Pienta KJ, Ben-Jacob E, Levine H: Coupling the modules of EMT and stemness: A tunable 'stemness window' model. Oncotarget 2015;6:25161-25174. 